\title{
Enhanced Buffer Router Design in NOC
}

\author{
Bhavana Prakash Shrivastava \\ Assistant Professor \\ Electronics Department \\ Maulana Azad Institute of Technology Bhopal India
}

\author{
Kavita Khare \\ Associate Professor \\ Electronics Department \\ Maulana Azad Institute of Technology Bhopal India
}

\begin{abstract}
This paper presents an advance router design using enhanced buffer. The design provides advantages of both buffer and bufferless network for that two cross bar switches are used. The concept of virtual channel (VC) is eliminated from the previous design by using an efficient flow-control scheme that uses the storage already present in pipelined channels in place of explicit input virtual channel buffers (VCBs). This can be addressed by providing enhanced buffers on the bufferless link and creating two virtual networks. With this approach, VCBs act as distributed FIFO buffers. Without VCBs or VCs, deadlock prevention is achieved by duplicating physical channels. An enhanced buffer provides a function of hand shaking by providing a ready valid handshake signal and two bit storage. Through this design the power is saving to $18.98 \%$ and delay is reduced by $99.13 \%$ as compared with the generic router and the power is saving to $15.65 \%$ and delay is reduced to $97.88 \%$ as compared to virtual channel router.
\end{abstract}

\section{General Terms}

Router design, Virtual channel, Buffer architecture, Virtual Channel Allocation.

\section{Keywords}

NOC, Gate delay, VCs, enhanced buffer, VCB.

\section{INTRODUCTION}

With the advancement in IC technology the gate delay decreases which leads to relatively increase the wire delay. The wire delay decides the overall performance of the system. Many VLSI designers are trying to solve this long global wire delay problem through buffer insertion. Many current System-on-Chips (SoCs) use a system bus to connect several functional units. These SoC system buses can support only limited number of functional units, and thus will face scaling problems in heterogeneous Multiprocessor System-on-Chips (MPSoCs) or large scale ChipMultiprocessors (CMPs). In order to solve these long global wire delay and scalability issues, many studies suggested the use of a packet based communication network which is known as Network-on-Chip (NoC). This NoC is used to connect many functional units with a universal communication network [1,2, 3]. In today's NoCs researchers are continuously confronted by several major challenges: reducing power dissipation in the network, improving performance etc.The power consumed by modern NoCs consists of a large portion of total chip power. For example, $28 \%$ of the total chip power of Intel Teraflops processors is spent for communication, while the expectation was only $10 \%$ [12]. Moreover, the interconnection network of MIT's RAW processor consumes $36 \%$ of the total chip power $[13,14]$. Power dissipation in modern NoC architectures is mainly characterized by the power consumed in the links, crossbars, and input buffers. Input buffers alone could consume up to $30-35 \%$ percent of the total power of the whole interconnection network [11]. As a result, reducing the size of input buffers or completely eliminating input buffers is a natural approach to design low-power NoCs. Simply reducing the size of input buffers in each router may result in a degraded performance such as reduced throughput etc. Different types of techniques have been proposed to reduce the size of input buffers or to eliminate them.

\subsection{Related Work}

To reduce or eliminate the size of input buffers, recently iDEAL (inter-router Dual-function Energy and Area-efficient Links) proposed to reduce the size of the input buffers and utilize repeaters with inter-router channels as storage units this design reduces the number of input buffer to half but with the cost of increased latency and complexity[13]. Other designs targeting power saving with router design have different approaches. A dynamic buffering resources allocation design named ViChaR (Virtual Channel Regulator) focuses on efficiently allocating buffers to all virtual channels, by deploying a unified buffering unit instead of a series of separated buffers, and minimizing the required size [7]. Bufferless routing is another novel and unique approach which eliminates all input buffers without utilizing channel buffering. Flit-Bless proposed a routing scheme to send all incoming packets to output ports, irrespective of the fact whether those output ports are productive [14]. The age-based priority for arbitration indicates that the oldest incoming packet is guaranteed to be routed to its productive output port, while younger packets may be deflected to their non-productive output ports and take non-minimal numbers of hops before reaching their destinations. In NoC, a router sends packets from a source to a destination router through several intermediate nodes. If the head of packet is blocked during data transmission, the router cannot transfer the packet any more. In order to remove the blocking problem, wormhole routing method is proposed in [4]. The wormhole router splits the packet into several flits which can be transferred in a single transmission. Buffer allocation and flit control are performed at a flit level in wormhole routing since wormhole routing does not allocate available buffer to whole packet [5]. Therefore, the wormhole routing is a method which can minimize overall latency and may decrease buffer size compared to others. In addition, VCs are used to avoid deadlock problem and thus increase throughput. The main purpose of VCs is to decouple the allocation of buffer space to allow a flit to use a single physical channel and competing with other flits. There are existing two router techniques based on wormhole and other based on virtual channel as discussed below. 


\subsubsection{Generic NOC Router}

The router implemented using wormhole is Generic NoC router shown in Figure 1.It has five inputs and output ports, each of which is for local processing element (PE) and four directions: North, South, West, and East. Each router also has five components: Routing Computation (RC) Unit, Virtual Channel Allocator (VA), Switch Allocator (SA), flit Buffers (BUF), and Crossbar Switch.

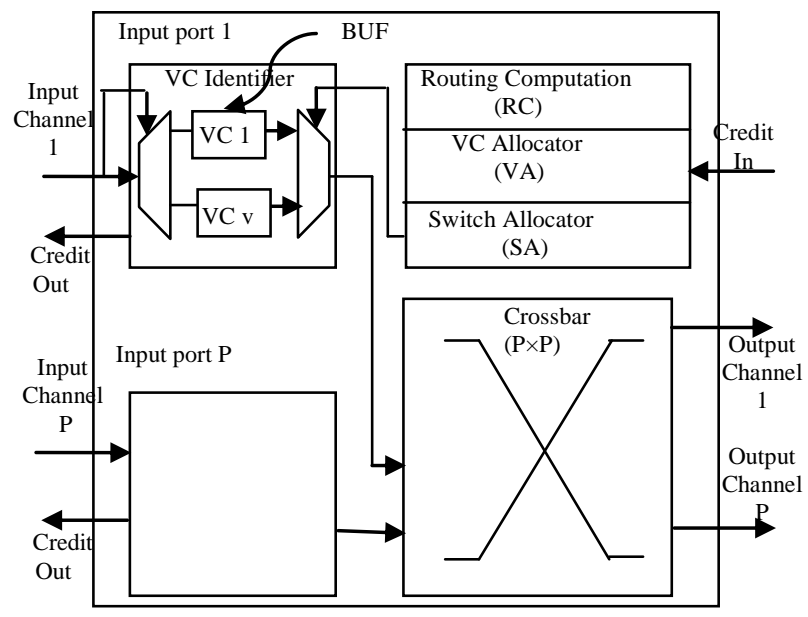

Figure 1: Generic NOC Router

When the header flit arrives at the buffer, the RC unit sends incoming flits to one of physical channels. The Virtual Channel Allocation(VA) unit receives the credit information from the neighboring routers, arbitrates all the header flits which access the same VCs, and then one of them was selected. Therefore, this header flit can set up the path and then send data. The transmitting router sends the control information to the receiving router, and receiving router may update VC information at the internal buffer with this control information. SA unit arbitrates the waiting flit in all VCs accessing the crossbar and allow only one flit to access crossbar .The SA operation is based on the VA stage since the flit data in the buffer comes from the previous router in the route. The flit data pass over the crossbar and thus can arrive at the destination node.

\subsubsection{Virtual Channel Regulator (ViChaR)}

The router implemented using virtual channel is ViChaR [7].This can efficiently allocating buffers to all virtual channels, instead of using separated buffers, a unified buffer structure (UBS) is used to share the internal flit buffers and Unified Control Logic (UCL,) to control UBS and assign buffers into VCs dynamically according to the network traffic [11]. Figures $2(\mathrm{a}, \mathrm{b})$ show the comparison between the ViChaR router buffer architecture with generic router buffer architecture. It is shown in the Figures 2(a,b) that UBS is similar to generic buffer structure in which the $\mathrm{v}$ independently $\mathrm{k}$ - flit are logically grouped in a single vk-flit and with UCL as logically unified structure. To avoid large components UBS has the same number of MUX/DEMUX i.e. one MUX/DEMUX per $\mathrm{k}$ flits. In the shown Figure 3(a) the first stage reduces the number of requests from each input $\mathrm{VC}$ to one and the winning request from each input $\mathrm{VC}$ proceeds to the second arbitration stage.

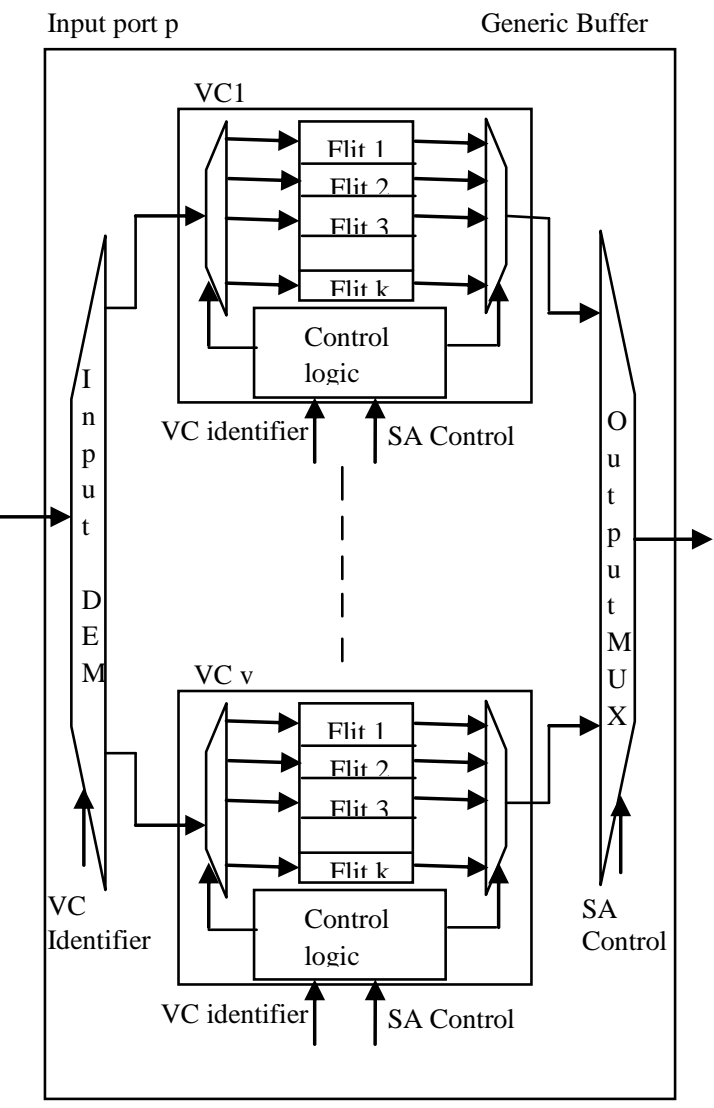

Figure 2 (a): Buffer architecture and allocation of generic router

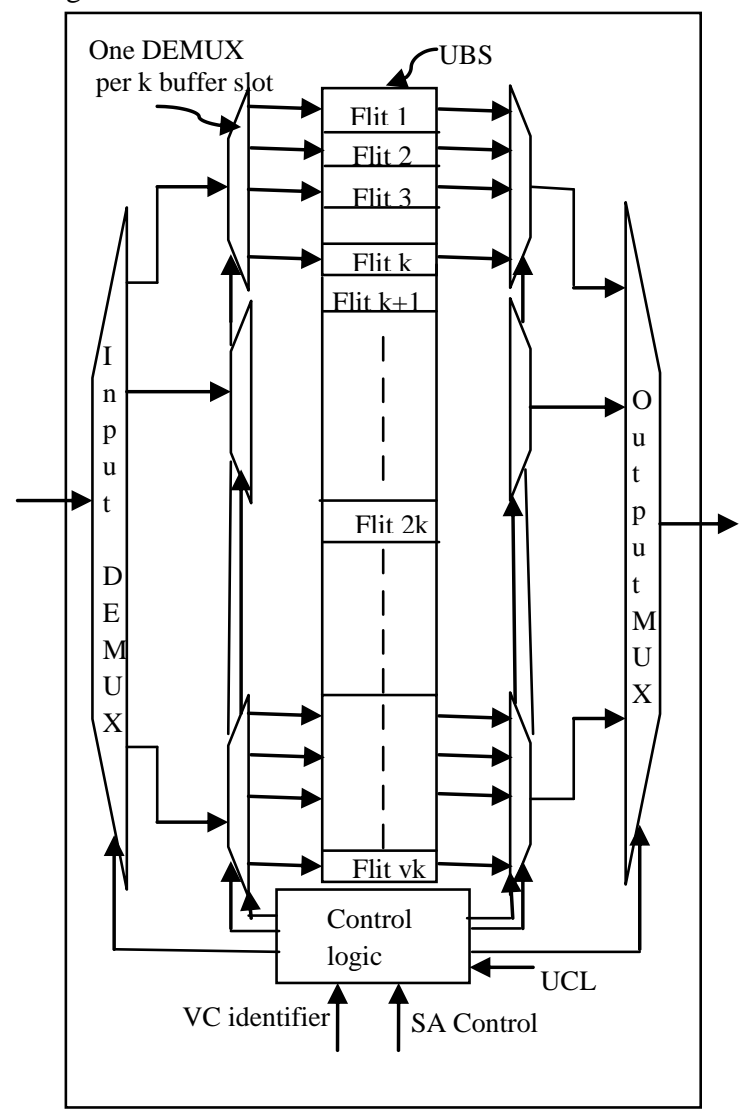

Figure2 (b): Buffer architecture and allocation of ViChaR router 
While in Figure 3(b) the first arbitration stage reduces the number of requests for a particular output port to one request per input port.In generic router implementation of VA first stage needs $\mathrm{v}$ : 1 arbiter as the number of VCs supported is fixed to $\mathrm{v}$ while in case of ViChaR the VA first stage needs vk:1 arbiter but in second stage a winner for each output port among all the competing input ports. So it just allocates the VCs instead of accepting requests for specific VCs.

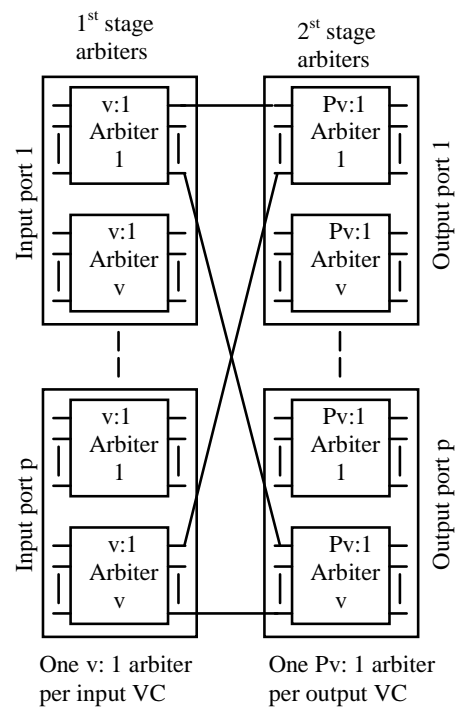

Figure 3(a): Virtual Channel Allocation for Generic case

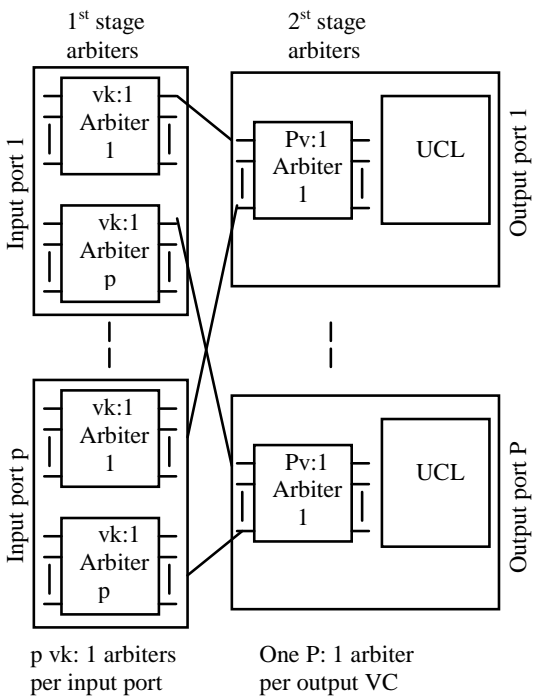

Figure 3(b): Virtual Channel Allocation for ViChaR Case

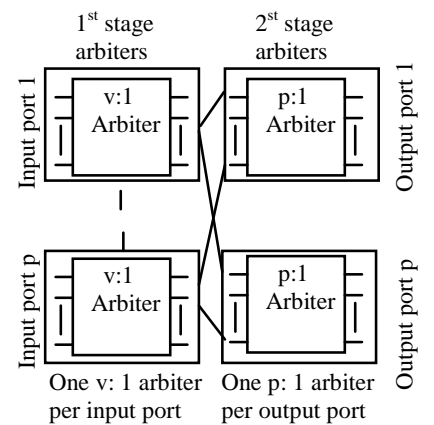

Figure 4(a): Switch Allocation for Generic case
Similarly the SA in Figure 4(a, b) the first stage of ViChaR sharing of a single port by a number of VCs, ViChaR needs larger $v k$ : 1arbiters. The second stage arbitrates between the winning requests from each input port (i.e. $P$ ports) for each output port; thus, it is the same for both architectures.

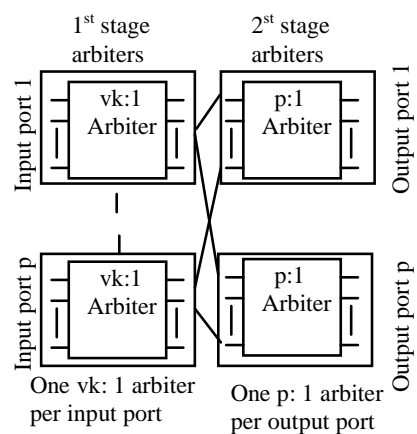

Figure 4(b): Switch Allocation for ViChaR Case

\section{PIPELINE STAGES}

NoCs routers are pipelined at the flit level to better utilize all the control units and improve the throughput. Figure: 6(a) shows the pipeline of a generic 6-stage router and timing of different flits in a packet .The stages are: buffer write (BW) Routing Computation (RC), Allocation (VA), Switch Allocation (SA), Switch Traversal (ST), and Link Traversal (LT).RC works when the head flit of a packet is stored in a virtual-channel, the routing information carried by the head flit is input to the router to determine the output port of the packet. Once the result is calculated, all of the flits in the same packet must use the same output port.VA works when the output port is determined, the result is input to the virtual-channel allocator to assign a single output virtual-channel on the corresponding output port. If the allocation fails, the head flit needs to wait until the output port has a free VC to assign. The allocation is performed for the head flit only. SA works when the output VC is assigned, per-packet operations are completed and switch allocation is performed flit-by-flit. All of the flits in a packet will consecutively bid for a single-flit time slot to traverse the switch.ST works when the switch is allocated to a flit, the flit uses one cycle to traverse the switch to the desired output port. LT works when the switch is traversed by a flit, the flit uses another cycle to traverse the channel and reach its downstream router. Each pipeline stage requires one cycle to perform. Therefore, a flit needs six cycles to traverse the router. In ViChaR buffer write (BW) and Routing Computation (RC) access simultaneously so the number of stage get reduce to 5 as shown in Figure:6(b). The problem which are concluded from the above two techniques is delay as processing time is too long and second due to the virtual channels the buffers are allotted to all VCs resulting in increased complexity in buffer management resulting increases power consumption $[7,10]$. So in order to overcome delay problem and power consumption problem new proposed router design come in to account so solve these problem, in the proposed design the Virtual channel is eliminated and the number of stages get reduced to three .

\section{PROPOSED METHODOLOGY}

In the proposed design the advantage of both bufferless and buffered networks in taken in to account for that two cross bar switches are used [9]. The proposed design is a combination of a bufferless primary crossbar and a buffered secondary crossbar. 


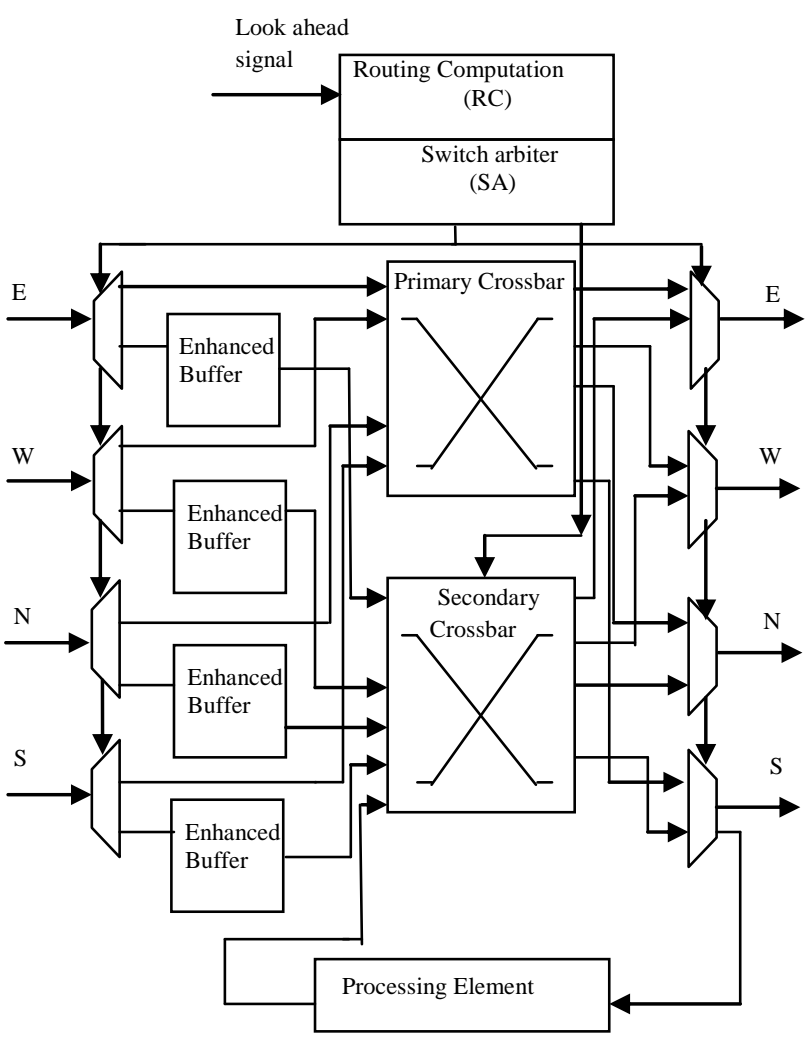

Figure: 5-Proposed router with enhanced buffer

At low load condition the all packets would only traverse the primary crossbar and follow minimum path and experiences minimum delay so behave as bufferless network. At high load the packets get traverse from secondary cross bar through a enhanced buffer which provides a function of hand shaking by providing a ready valid handshake signal. In the Figure 5 proposed router with enhanced buffer is shown. It is having four input port at low traffic the data flit traverse though primary switch at heavy load the flit get store in the enhanced buffer and the data flit traverse through secondary crossbar. The function of processing element is to give feedback from output to input to show whether the flit is valid or not. Buffers are provided in front of secondary in which the data moves serially as the virtual channel is eliminated so the virtual allocator stage is eliminated. Switch arbiter (SA) is modified to make control over the Demux and Mux to maintain the correct packet flow in both crossbars. The elimination of VCs eliminates the VA stage and simplifies SA stage, so that SA and ST could be performed in the same cycle the number of stages reduce to 3 so shown in Figure 6(c).

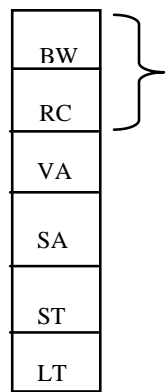

(a)

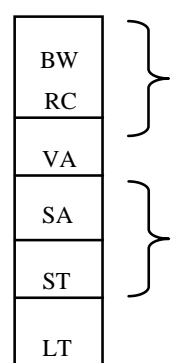

(b)

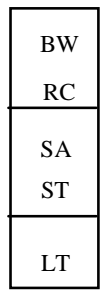

(c)
Figure 6: (a) 6 stage generic router (b) 5 stage ViChaR router(c) 3 stage proposed router with enhanced buffer
The function of enhanced buffers is a flow-control scheme that uses the storage already present in pipelined channels in place of explicit input VCBs. With this approach, the channels themselves act as distributed FIFO buffers under congestion. Without VCBs, and hence VCs, deadlock prevention is achieved by duplicating physical channels. Duplicate physical channels must be used in the same way as VCs to prevent deadlocks and to differentiate between traffic classes. Figure 7(a) shows a Dflip-flop (DFF) that is implemented using master and slave latches. By adding control logic to drive the latch enable pins independently, each latch can be used as an independent storage location. Thus, the FF becomes an EB, a FIFO with two storage locations. This is illustrated in Figure 7(b).

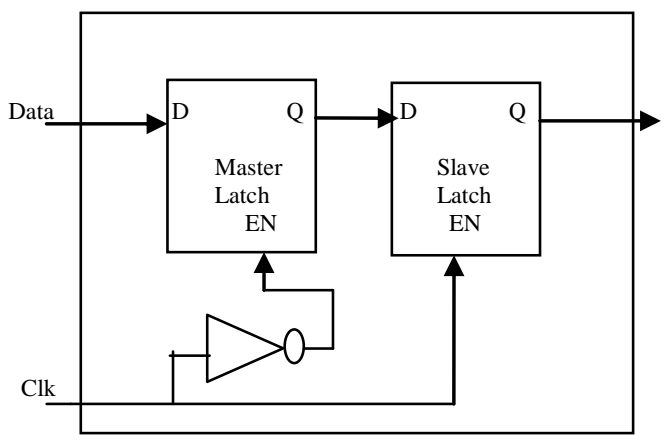

Figure: 7 (a) A DFF with master and slave D latch

EB use ready(R) and valid (V) handshake to advance data. Output of ready indicates that enhanced buffer has at least one empty storage slot to latch more data. Valid outputs indicate that the data currently being driven is valid. The control logic samples incoming control signals at rising clock edges to determine if data has advanced to or from the Enhanced buffer. Asserted ready and valid signals between two EBs indicate that data has advanced. Data is latched in the master latch at the end of the cycle, and advances to the slave at the beginning of the next cycle. . If during the previous cycle the incoming ready was de-asserted and the state remains at 1 , data is already in the slave latch and its enable input needs to be disabled .In the buffered secondary crossbar the incoming

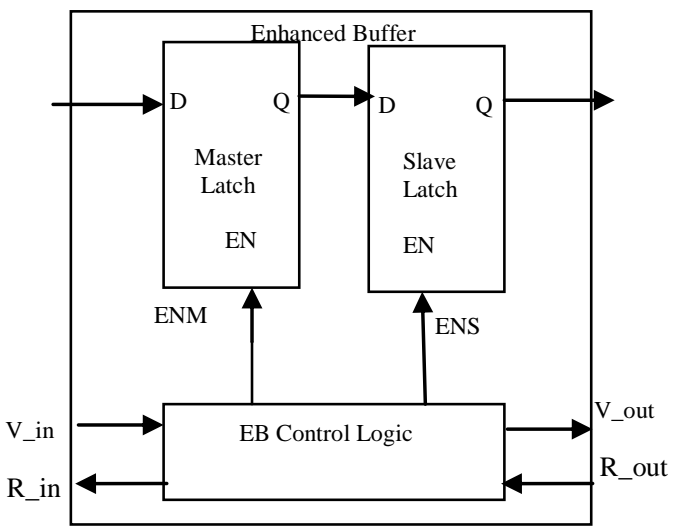

Figure 7 (b): A EB is a FF whose latch are control by the EB control logic

flits are stored at the selected cross point for their output. At the second stage, outputs arbitrate among all the valid cross points and store flits into the output. The ready-valid handshake is used 
in both stages. The comparison of three techniques is shown in the Table 1 based on the parameters.

Table 1: Comparison of proposed router with other on the basis of following parameters

\begin{tabular}{|c|c|c|c|}
\hline Parameters & $\begin{array}{l}\text { Generic } \\
\text { router }\end{array}$ & $\begin{array}{l}\text { Virtual } \\
\text { channel } \\
\text { router }\end{array}$ & $\begin{array}{l}\text { Proposed } \\
\text { enhanced } \\
\text { buffer } \\
\text { router }\end{array}$ \\
\hline $\begin{array}{l}\text { Buffered/ } \\
\text { Buffer less }\end{array}$ & Buffered & Buffered & $\begin{array}{c}\text { Both } \\
\text { buffered } \\
\text { and } \\
\text { buffereless }\end{array}$ \\
\hline $\begin{array}{c}\text { Number of } \\
\text { crossbar switch }\end{array}$ & One & One & Two \\
\hline Type of Buffer & $\begin{array}{l}\text { Simple } \\
\text { Buffer }\end{array}$ & $\begin{array}{c}\text { Virtual } \\
\text { channel as } \\
\text { buffer }\end{array}$ & $\begin{array}{c}\text { Enhanced } \\
\text { buffer }\end{array}$ \\
\hline $\begin{array}{c}\text { Number of } \\
\text { processing } \\
\text { steps }\end{array}$ & Six & Five & Three \\
\hline $\begin{array}{c}\text { Probability of } \\
\text { data loss }\end{array}$ & High & Medium & $\begin{array}{c}\text { Low or } \\
\text { Negligible }\end{array}$ \\
\hline $\begin{array}{l}\text { Hand shaking } \\
\text { phenomenon }\end{array}$ & No & No & Yes \\
\hline
\end{tabular}

As shown in the Table 1 we can see that the advantage of both buffer as well as bufferless is achieved. Enhanced buffer is used which is based on control logic. The number of processing step is reduced to three as the virtual channel allocator (VA) is removed. The probability of data loss is low or negligible as it works on the concept of handshaking which works on the availability of valid or ready signal till the no flit is transmit or received. Based on the above parameter proposed enhanced buffer router give better performance compare to other techniques.

\section{IMPLEMENTATION AND RESULTS}

Implementation is done in cadence virtuoso at $180 \mathrm{~nm}$.The designing parameter are selected at the time of designing. Generic router and virtual channel router are design for making comparative study between the three techniques. Table 2 shows the comparison based on the delay and total average power.

Table 2: Comparison with the existing techniques

\begin{tabular}{|l|c|c|}
\hline Design & Delay(nsec) & $\begin{array}{l}\text { Total average } \\
\text { power }(\mu \mathrm{W})\end{array}$ \\
\hline Generic router & 41.39 & 527.7 \\
\hline $\begin{array}{l}\text { Virtual channel } \\
\text { router }\end{array}$ & 0.877 & 445.1 \\
\hline $\begin{array}{l}\text { Proposed } \\
\text { enhanced buffer } \\
\text { router }\end{array}$ & 0.358 & 427.5 \\
\hline
\end{tabular}

The delay and average power of generic router is maximum, which are significantly reduced using virtual channel router. In proposed design both the quantities reduced further as we can also see in Figure 8 and Figure 9.

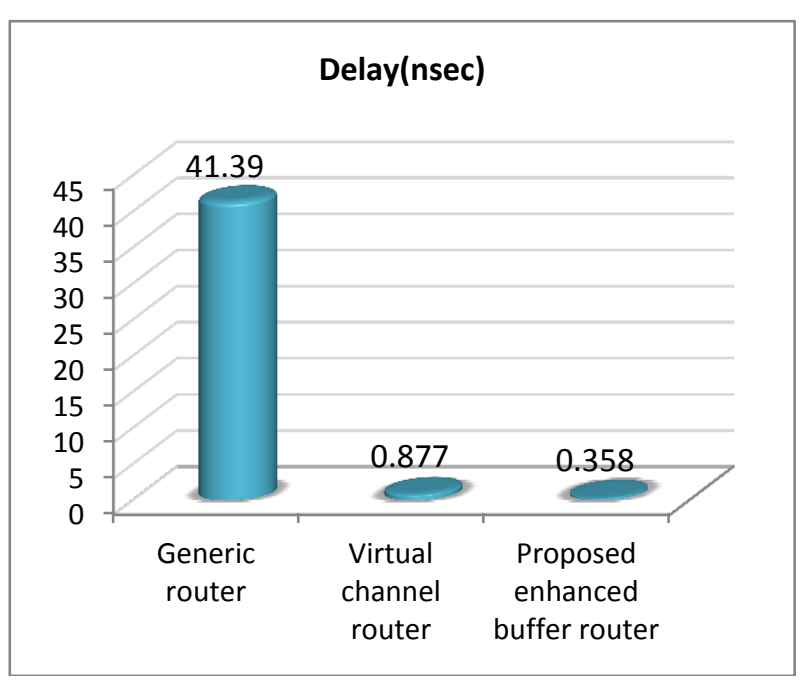

Figure 8: Delay in different routers

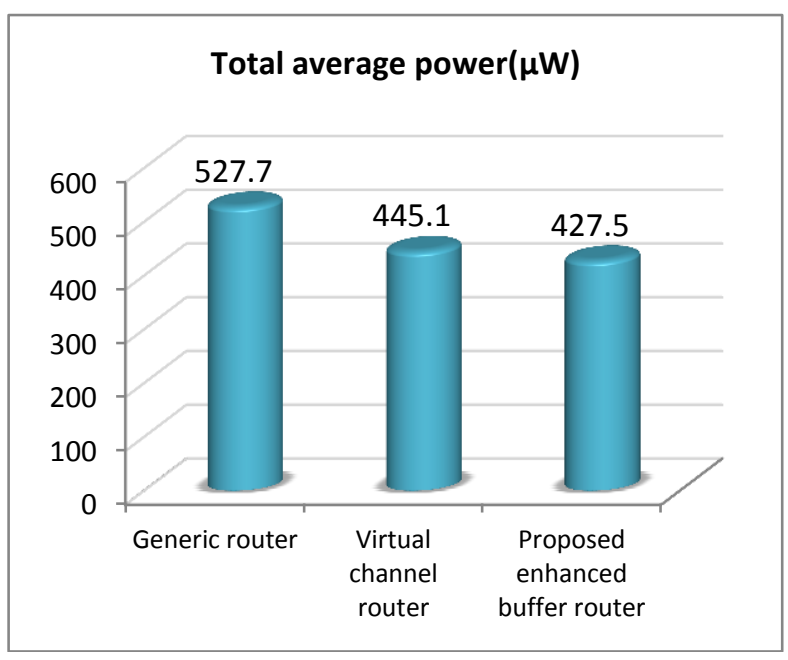

Figure 9: Total average power in different routers

Table 3 shows the percentage reduction of delay and power of virtual channel and proposed router in terms of delay and power. The virtual channel router results $97.8811 \%$ reduction in delay and $15.6528 \%$ saving in average power as compared to generic router. The proposed enhanced buffer router performs better then virtual channel router in both the parameters. it diminishes the delay by $99.1350 \%$ and average power by $18.9881 \%$ as that of generic router. Figure 10 shows the circuit diagram of proposed enhanced buffer Router. Figure 11 show the delay of proposed enhanced buffer Router. Figure 12 show the power of proposed enhanced buffer Router. 
Table3: Percentage change reduction with generic router

\begin{tabular}{|l|c|c|}
\hline Design & $\begin{array}{l}\text { \% change in } \\
\text { Delay w.r.t. } \\
\text { generic router }\end{array}$ & $\begin{array}{l}\text { \% change in Total } \\
\text { average power w.r.t. } \\
\text { generic router }\end{array}$ \\
\hline $\begin{array}{l}\text { Virtual } \\
\text { channel router }\end{array}$ & 97.8811 & 15.6528 \\
\hline $\begin{array}{l}\text { Proposed } \\
\text { enhanced } \\
\text { buffer router }\end{array}$ & 99.1350 & 18.9881 \\
\hline
\end{tabular}

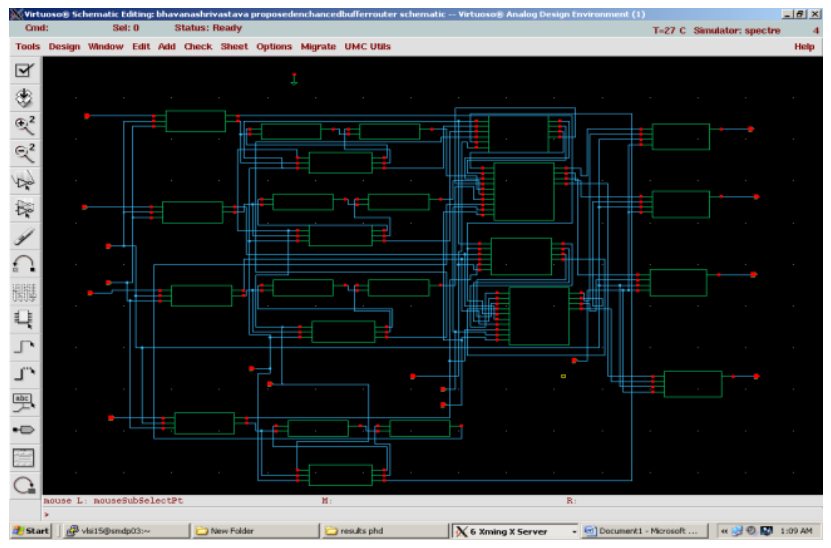

Figure 10: Proposed enhanced buffer Router Circuit

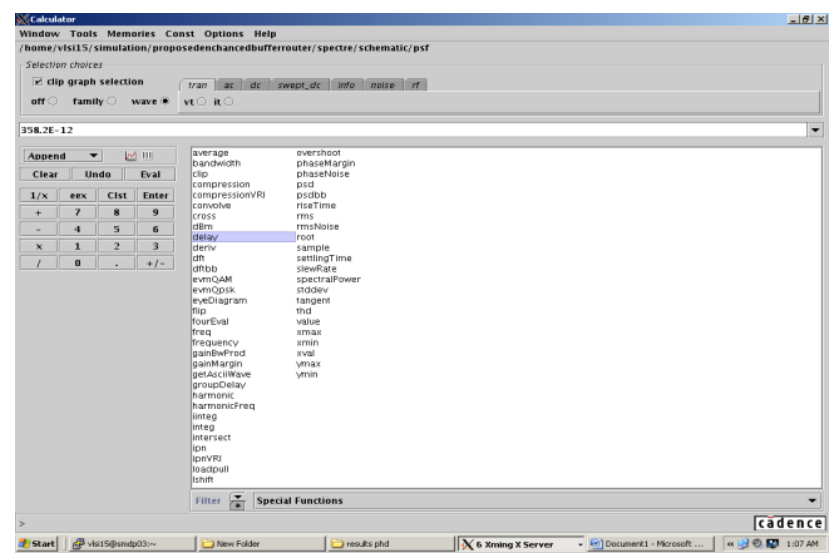

Figure 11: Delay of proposed enhanced buffer Router

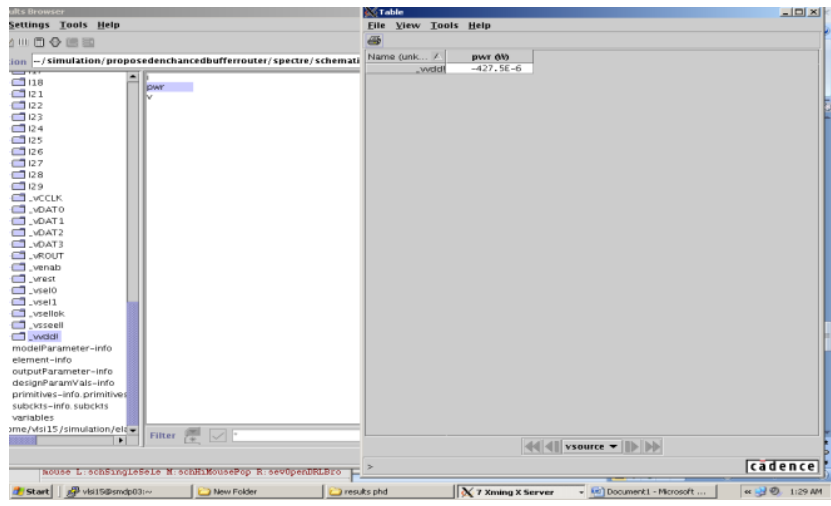

Figure 12: Power of proposed enhanced buffer Router

\section{CONCLUSION}

In proposed design the advantage of both buffered and bufferless is achieved. At low load condition the packet traversal is done through a bufferless network and at high load the packet traversal is through enhanced buffer ie buffered network. It provides better results than generic NoC router as well as virtual channel router. Proposed router achieves $18.98 \%$ power saving compared to generic router and $15.6 \%$ compare to virtual channel router. In terms of delay the proposed router provided $99.13 \%$ less delay than generic router and $97.88 \%$ less delay as compared to virtual channel router. Reducing pipeline stages result in reduced delay for packets in each router, which in turn decreases the average latency. Because packets spend less time traveling in the network, the now-unoccupied cycles enable more packets to be transmitted and increase the throughput of the network.

\section{REFERENCES}

[1] L. Benini and G. De Micheli, "Networks on chips: A new SoC paradigm,"Computer, vol. 35, no. 1, pp. 70-78, 2002.

[2] W. J. Dally and B. Towles, "Route packets, not wires: Onchip interconnection networks," in DAC '01: Proceedings of the $38^{\text {th }}$ Conference on Design Automation, Jun. 2001, pp. 684-689.

[3] P. Guerrier and A. Greiner, "A generic architecture for onchip packet-switched interconnections," in DATE '00: Proceedings of the Conference on Design, Automation and Test in Europe, Mar. 2000, pp. 250-256.

[4] Z. Lu and A. Jantsch, "Flit ejection in on-chip wormholeswitched Networks with virtual channels," in NORCHIP '04: Proceedings of the 2004 IEEE/ACM International Conference on Norchip, Nov. 2004, pp. 273-276.

[5] J. Hu, “ U. Y. Ogras, and R. Marculescu, "System-level buffer allocation for application-specific networks-on-chip router design," IEEE Trans. on CAD of Integrated Circuits and Systems, vol. 25, no. 12, pp. 2919-2933, Jan. 2006.

[6] Z. Lu and A. Jantsch, "Flit ejection in on-chip wormholeswitched Networks with virtual channels," in NORCHIP '04: Proceedingsof the 2004 IEEE/ACM International Conference on Norchip, Nov. 2004, pp. 273-276.

[7] C. A. Nicopoulos, D. Park, J. Kim, N. Vijaykrishnan, M. S. Yousif, and C. R. Das, "ViChaR: A dynamic virtual channel regulator for network-on-chip routers," in MICRO'39: Proceedings of the 39th Annual IEEE/ACM International Sympo-sium on Microarchitecture, Dec. 2006, pp. 333-346.

[8] L. S. Peh, W. J. Dally, and P. Li-Shiuan, "Delay model for router microarchitectures," IEEE Micro, vol. 21, no. 1, pp. 26-34, 2001.

[9] T. Moscibroda, O. Mutlu, "A case for bufferless routing in on-chip networks, in Proceedings of the 36th Annual International Symposium on Computer Architecture, June 2007.

[10] S. Borkar, "Design challenges of technology scaling," IEEE Micro, vol. 19, pp. 23-29, 1999

[11] S. Ramany and D. Eager, "The interaction between virtual channel flow control and adaptive routing in wormhole networks," in ICS '94: Proceedings of the $8^{\text {th }}$ International Conference on Supercomputing, Jul. 1994, pp. 136-145. 
[12] Y. Hoskote, S. Vangal, A. Singe, N. Borkar, and S. Borkar, "A 5-ghz mesh interconnect for a teraflops processor," IEEE Micro, vol. 27, no. 5, 2007.

[13] H. Wang, L.-S. Peh, and S. Malik, "Power-driven design of router

[14] Microarchitectures in on-chip networks," in MICRO 36: Proceedings of the 36th annual IEEE/ACM International Symposium on Microarchitecture. Washington, DC, USA: IEEE Computer Society, 2003, p. 105.

[15] S. Kim, M. B. Taylor, J. Miller, and D. Wentzlaff, "Energy characterization of a tiled architecture processor with on- chip networks," in ISLPED '03: Proceedings of the 2003 international symposium on Low power electronics and design. New York, NY, USA: ACM, 2003, pp. 424-427.

[16] A. K. Kodi, A. Sarathy, and A. Louri, "ideal: Inter-router dual-function energy- and area-efficient links for networkon-chip (noc)," in Proceedings of the 35th International Symposium on Computer Architecture.(ISCA'08), Beijing, China, June 2008, pp. 241-250.

[17] T. Moscibroda and O. Mutlu, "A case for bufferless routing in on-chip networks," in Proceedings of the 36th annual International Symposium on Computer Architecture, June 2007. 